\title{
EI TRABAJO SOCIAL EN LAS INSTITUCIONES CARCELARIAS PARA MUJERES: IMPLICACIONES PARA LA PRÁCTICA
}

\author{
Blanca E. Hernández Sierra ${ }^{I}$
}

\section{Resumen}

Los servicios sociales en instituciones carcelarias están limitados por la filosofía del control y castigo que rige en muchas de estas. Ello representa retos para la labor de los (las) profesionales de trabajo social que fundamentan su práctica en los valores de la justicia social y en la convicción de que los seres humanos son capaces de dirigir sus acciones por la solidaridad y el compromiso con ellos mismos y con otros. Este trabajo discute las percepciones contradictorias sobre la mujer confinada que subyace el ofrecimiento de los servicios sociales y sus implicaciones para la práctica, tomando como base un estudio de evaluación de los servicios sociales que se ofrecen en la Escuela Industrial para Mujeres en Vega Alta. Sobresale en la discusión, la percepción que tienen las(os) participantes del estudio, de su rol profesional en la institución, así como las condiciones de trabajo que le condicionan la práctica.

Descriptores: mujer confinada, percepción de la mujer confinada, sistema carcelario, servicios socio-penales, servicios sociales.

\begin{abstract}
Social services in prison settings are limited by the philosophy of control and punishment that exists in many of these institutions. This represents a challenge to the practice of social work based on the value of social justice and the valued principal of dignity of all human beings. This paper discusses the conflicting perceptions about women in prison, and the implications for the social work practice. The work presented is the result of a program evaluation study on social services offered in the correctional institution for women in Vega Alta, Puerto Rico. The work also discusses the perception of participants in the study, of their professional role in the institution, as well as the working conditions which limits their practice.
\end{abstract}

${ }^{1}$ Catedrática Escuela Graduada de Trabajo Social Beatriz Lassalle, Universidad de Puerto Rico en Río Piedras 
Key words: women in prison, perception of women in prison, prison system, social services in prison settings.

\section{Introducción}

La Escuela Industrial para Mujeres de Vega Alta fue creada por virtud de la Ley Núm. 422 del 23 de abril de 1946. Se incorpora como institución penal al programa de Corrección del Departamento de Justicia, el 16 de agosto de 1954 (Peña Beltrán, 1986). Esta Institución se crea bajo la disposición legal que indica que:

....funcionará bajo el régimen tendiente a la rehabilitación social, física y moral de las reclusas, según lo amerite cada caso, proporcionándoles instrucción escolar e industrial para que, al restituirse aquéllas a la sociedad, después de extinguida su sentencia, puedan sostenerse y ser útiles a su familia y a la comunidad (Peña Beltrán, 1986, pág. 85).

De acuerdo con Peña Beltrán (1986), primera Directora de la Escuela Industrial, el primer grupo de mujeres confinadas tenía un perfil que correspondía a una mujer de poca preparación académica, con pocas destrezas ocupacionales, que no le permitían participar en el mundo laboral asalariado. Muchas de ellas habían vivido del oficio de la prostitución por varios años y se adaptaban fácilmente a las normas institucionales y a su disciplina. Peña Beltrán (1986) en sus observaciones señala, que estas mujeres llegaban a la Institución con el objetivo de cumplir y regresar a sus comunidades y esto se observaba en la frase de las mismas confinadas: "Lo mío no es perpetua." Esta frase es interesante porque nos permite concluir, que las mujeres veían en la Institución un estado de vida transitorio, lo cual implica que no existía una identidad de adaptación institucional. Este hecho es de suma importancia, ya que nos muestra que el confinamiento era percibido como una condición de vida temporal en la institución. Por ello, Peña Beltrán (1986) concluye que:

....la filosofía de tratamiento al delincuente ... debería partir casualmente de esa premisa, o sea, de que el tiempo de reclusión de un individuo es de carácter transitorio y por lo tanto, la Institución deberá tener objetivos inmediatos y objetivos a largo alcance para preparar mejor a ese individuo para su retorno a la sociedad (pág.87).

\section{La mujer en el sistema carcelario}

Como consecuencia de la guerra contra las drogas y la lucha contra el crimen, la población penal en Estados Unidos ha aumentado (Phillips y Harm, 1998). En Puerto Rico esta situación es similar debido en gran medida, a la adopción de las mismas estrategias legales y la política social para combatir las drogas y la criminalidad en la Isla (González, Márquez y Márquez, 2003). De acuerdo con la Exposición de Motivos 
del proyecto de la Cámara de Representantes 130 del 22 de enero de 1997 para crear una Comisión Especial que realice un estudio abarcador sobre el creciente problema de la criminalidad, la alta incidencia de violencia y el rampante trasiego de drogas ilícita. La incidencia criminal en la Isla se ha ligado estrechamente al imperante tráfico de drogas ilícitas. Con ello se menciona un aumento en la población confinada de la Isla.

Según datos oficiales disponibles del Departamento de Corrección y Rehabilitación para el 2003 había un total de 12, 781 confinados en las cárceles de Puerto Rico. De igual forma, la presencia de la mujer en el sistema carcelario de Puerto Rico se hace evidente con la inauguración de la Escuela Industrial para Mujeres en Vega Alta. Desde su inauguración en 1954 hasta 1964, la Institución atendió un promedio de 95 a 110 confinadas, cifra que aumentó en 1970 a 169 (Picó, 1994). Según Pico, esta cifra se elevó en la década de los ochenta. Esta situación coincide con la política de la guerra contra las drogas que se inició oficialmente, con el acta federal de América Libre de Drogas en 1987. Correspondiendo con este proceso y con algunas fluctuaciones mínimas en las estadísticas, la presencia de la mujer en el sistema correccional de Puerto Rico, refleja un total de población confinada relativamente constante. Iniciando con el 8 de junio 1993, podemos señalar que los informes oficiales del Perfil de la Confinada, reflejaban una población de mujeres confinadas que alcanzaba la cifra de 300 (Rose and Associates, n/d). Una década después, al 30 de junio de 2003 el perfil oficial de la mujer confinada informó una población total de 291, lo que refleja un leve descenso que se vuelve a revertir en junio del 2004 con una población total de 386. En el 2005 se refleja un leve descenso para una cifra de 359 (Departamento de Corrección y Rehabilitación, 2005) y en el 2008 el informe indicaba que al 1 de julio de ese año la población confinada sentenciada era de 277.

A pesar de que la presencia de las mujeres en el sistema correccional se ha hecho evidente, las necesidades de éstas se han visto opacadas por la preocupación hacia la población masculina, que continua predominando en las cárceles (Harden y Hill, 1998). Históricamente el sistema correccional ha respondido a la mujer ofensora sobre la base de estereotipos de ésta como incapaz de rehabilitarse. También se le ha visto como necesitada de adiestramiento en ocupaciones tradicionalmente femeninas (Peña Beltrán, 1986), así como un riesgo sustancial a la integridad moral masculina, lo cual limita el acceso igual a los mismos programas (Harden y Hill, 1998). Por otro lado, no sólo la necesidad de igualdad programática para ayudar a estas mujeres se ha visto opacada sino, la diferencia en los servicios que requieren las confinadas. De acuerdo con estas autoras, estas diferencias no se han podido atender, porque no se ha hecho la conexión entre la criminalidad cometida por las mujeres, el contexto en el cual ésta se produce, así como los procesos de arresto y encarcelamiento asociados. En Puerto Rico, la encarcelación se da predominantemente, entre las mujeres pobres, adictas y sobrevivientes de abuso sexual y físico (Departamento de Corrección y Rehabilitación, 2005). 
Por lo antes expuesto, concurrimos con Harden y Hill (1998) en que las mujeres confinadas necesitan desarrollar destrezas educacionales y vocacionales que sean mercadeables, un sistema de apoyo familiar y social que les permita fortalecer una percepción de sí mismas que les dé crédito por sus fortalezas y recursos. Sobre todo, necesitan estrategias que les ayude a reconocer que lo que pasa en sus vidas personales es parte de las estructuras de poder que se configuran en la sociedad en general (1998). Esto es particularmente importante en las situaciones de las confinadas que han sido víctimas de violación y de maltrato. También se necesita atender los asuntos familiares, ya que la familia misma se encuentra atrapada en la dinámica pre-carcelaria (1998). Sobre todo, porque es la familia la mayor fuente de apoyo moral, social, económico y psicológico de estas mujeres, durante y después del encarcelamiento (González, Márquez y Márquez, 2003).

En el marco de los servicios sociales que se les ofrece a las confinadas, Coggins y Fresquez (2001) señalan que profesionales de trabajo social y otro personal de los servicios socio-penales que laboran en las instituciones correccionales, deben ser profesionales con amplias destrezas que se aparten del foco limitado de la penalización, al atender los problemas tan variados que esta población suele presentar. Entre las destrezas necesarias para atender adecuadamente a la población correccional, está la intervención en crisis y la competencia cultural para entender cómo la confinada percibe su experiencia carcelaria. También necesitan habilidad para entender y recabar la coordinación con otras agencias, así como otros recursos en la comunidad. Además de los conocimientos para la intervención con la confinada, este personal necesita conocimientos de la práctica a nivel macro, ya que los múltiples sistemas fuera de la Institución, pueden afectar grandemente a las confinadas, tanto en el proceso de ingreso, como en la estadía y la salida de la cárcel. De la misma forma, Coggins y Fresquez (2001) señalan la importancia de poseer algún conocimiento en aspectos gerenciales que les permita evaluar la estructura correccional y su ambiente, a fin de contribuir al esfuerzo de mejorar el funcionamiento de la Institución.

Más importante aún, Coggins y Fresquez (2001) recomiendan, que las trabajadoras y trabajadores sociales que ofrecen servicios en instituciones penales, deben tener un sentido profesional sólido y claro, ya que éstos son ambientes complejos donde la labor del trabajo social constituye un servicio de apoyo y no un fin principal, lo que puede crear confusión en sus roles. Para estos autores, las cárceles son ambientes que producen altos niveles de tensión y aquellos empleados que no pueden manejar esta situación, no duran mucho en sus puestos. Un aspecto que contribuye a crear tensión, es la necesidad de atender los reclamos impuestos por la meta institucional de control y las contradicciones que surgen al atender las necesidades particulares de las confinadas. Esta capacidad de trabajar en ambientes de gran tensión es sobre todo importante, porque los servicios sociales que se ofrecen, se dan en un contexto donde cada cual tiene que utilizar estrategias diversas, incluyendo las de manipulación, para poder sobrevivir en un ambiente de reto y hasta hostil para todas las partes. Aún en las 
relaciones con otros trabajadores y trabajadoras, aquellos (as) profesionales que ofrecen los servicios sociales en la Institución se les hace difícil que otros entiendan lo que es la confidencialidad con la confinada y acorde con ello, la necesidad de guiarse por los códigos de ética de la profesión. Por ello, se concurre con Coggins y Fresquez (2001) cuando establecen, que un punto de convergencia entre los distintos actores en las instituciones penales, debe ser la meta general de que los servicios que se ofrecen estén orientados por la realidad de que la mayoría de las personas confinadas, retornarán algún día a la comunidad. Para ello, la meta de los servicios profesionales debe ser, el facilitar este proceso de reintegración.

\section{Servicio social y trabajo social en la Escuela Industrial para Mujeres de Vega Alta: Evaluación de programa}

Los argumentos discutidos anteriormente en torno al rol de profesionales que laboran el instituciones carcelarias, en especial el de trabajadoras y trabajadores sociales, nos lleva al análisis de los hallazgos de los resultados de una investigación finalizada en el 2003 sobre el Perfil de la Mujer Confinada, auspiciada por el Instituto FILIUS (Institute of Correction and Rehabilitation Central Administration). Este estudio incluyó un proyecto de evaluación de los servicios sociales que se les ofrece a las mujeres confinadas, en los programas de Salud Correccional (SC) y Servicios Socio- penales (SSP), mediante el uso de grupos focales y la revisión de expedientes. Estos hallazgos nos sirven de referencia para evaluar la propuesta señalada por Peña Beltrán (1989) y Coggins y Fresquez (2001) para que se considere como meta oficial, el que la mayoría de las mujeres confinadas retornaran algún día a la comunidad y por ello validar el que la meta de los servicios profesionales deben ser, el facilitar este proceso de reintegración.

La discusión en los grupos focales estuvo dirigida por las siguientes preguntas:

¿Cuáles considera usted que son las necesidades más apremiantes de las mujeres encarceladas en esta Institución?: personal, familiar, social, legal, otras.

¿Qué servicios y actividades les debe ofrecer la Institución (o el sistema de corrección) a estas mujeres, para que una vez excarceladas no reincidan?

¿Cuál debe ser la función del trabajador(a) social en el plan de ayuda psicosocial, para lograr que estas mujeres no reincidan?

\section{Percepción de la mujer confinada:}

Como nos señala Harden y Hill (1998), históricamente el sistema correccional ha respondido a la mujer ofensora sobre la base de estereotipos de ésta como incapaz de 
rehabilitarse. Por ello la percepción que se tiene de la mujer confinada es central en la prestación de los servicios y marco referencial para el análisis de los hallazgos de este estudio. Este tema surge tanto de la discusión en los grupos focales, como en la revisión de expedientes de las confinadas en la Escuela Industrial para Mujeres de Vega Alta y se considera fundamental para el análisis aquí presentado. Conceptualmente las percepciones integran visiones de mundo que no solo guían las acciones personales y profesionales, sino también las dinámicas inter e intra agénciales. La visión de mundo se considera:

....la ventana conceptual, a través de la cual percibimos e interpretamos el mundo, tanto para comprenderlo como para transformarlos. Esta ventana funciona como una especie de lente cultural, donde los ingredientes para su construcción incluyen valores, creencias, principios, premisas, conceptos, enfoques, etc., que moldean nuestra percepción de la realidad y, por lo tanto, nuestras decisiones y acciones hacia todos los aspectos de nuestra experiencia humana en el universo (Mato Bode et al., 2001, pág.10).

Aunque en los temas presentados para discusión en los grupos focales, no se incluyó la pregunta específica sobre la percepción que tienen las y los participantes profesionales en relación con la confinada, en la dinámica de la discusión se confrontaron visiones diferentes asociadas a la mujer transgresora, entre el personal de los distintos programas. El personal de Salud Correccional percibe a la confinada como una mujer necesitada de ayuda y servicios. El personal de Servicios Socio-penales la percibe como una transgresora de la ley con poca posibilidad de rehabilitación. Esta visión en particular considera que:

La población de mujeres es distinta a la de los hombres que buscan progresar. Las mujeres son más ansiosas, más depresivas. Contrario a los hombres, las mujeres todo lo dicen y por otra parte la mujer cuenta con más recursos de apoyo, ya que alguien siempre se acuerda de ellas. Un por ciento muy pequeño de las mujeres logran rehabilitarse. Hay sus excepciones, pero la mayoría no desea rehabilitarse (SSP).

El poco potencial de rehabilitación que se les atribuye a estas mujeres, se centra en la noción discriminatoria de que ella es la responsable de sus problemas legales. Se niega por tanto, la influencia de las circunstancias personales, sociales y económicas de marginación y opresión que sufren muchas de estas: El personal de Servicios Sociopenales expresa: "Llegan bien deterioradas, porque el sistema agota todos los recursos antes de confinarlas".

Esa percepción se presenta en la literatura como un conocimiento popular que se ha dado por cierto, aún en los círculos académicos y profesionales (Smart, 1995). La misma se enmarca en una concepción de determinismo biológico, donde se estipula 
que el temperamento, la inteligencia, la habilidad y la agresión de la mujer, están predispuestos por su condición física. Por ello se sostiene que los cambios hormonales, la menstruación entre otras, son causa de la conducta en la mujer, incluyendo la conducta criminal. De la misma forma Smart (1995), en sus ensayos sobre mujer y criminalidad, nos indica que otra concepción popular es que las mujeres cuando comenten delitos son más crueles y violentas. En contraste se considera que los hombres son más racionales que las mujeres. Las mujeres cuando cometen delito están enfermas (locas) y necesitan tratamiento psiquiátrico.

De forma similar, la revisión de los expedientes de las confinadas nos muestra, que los mismos no recogen información que permita atender las situaciones sociales, emocionales y económicas para un plan de acción dirigido a preparar a la mujer para su regreso a la comunidad. No existe información sobre patrones de violencia doméstica, ni de sus relaciones de pareja, a menos que estas no se relacionen directamente con el delito cometido. El plan de ayuda o servicio tampoco va dirigido a atender necesidades como el maltrato y abuso sexual, los patrones de violencia que conforman las experiencias de vida de estas mujeres, incluyendo la violencia familiar y comunal. Por su parte, los datos biográficos específicos que se recogen en el expediente, reflejan la construcción de un delincuente varón y contiene categorías de rasgos y apariencia física que se refiere a un hombre, como por ejemplo: tiene barba. Esto indica que el expediente que se utiliza en la institución para mujeres, es una réplica del expedientes socio-penal desarrollado para la población confinada de varones. Por ello la perspectiva de género está ausente y como consecuencia, el plan de servicios no refleja la información necesaria para atender las necesidades de la mujer confinada.

Enmarcada en esta visión de la mujer confinada, están los conceptos de control y castigo. La noción de control va dirigida a la visión de la confinada como un ser inmaduro que carece de auto-controles y como consecuencia la noción del castigo como estrategia de rehabilitación, es central a la filosofía carcelaria (la mujer niña que necesita tutelaje). Por esta razón, la discusión en el grupo focal de empleadas socio penales se trajo con frecuencia que la institución es un sistema de control carcelaric y ello era pertinente para la definición de sus funciones, encaminadas a facilitar la adaptación de la confinada a la vida institucional. Esta aseveración permite inferir que la orientación en los servicios que ofrecen las oficiales socio- penales se dirige a atender las necesidades de las confinadas dentro de la Institución sin consideración de que la meta debe ser prepararlas para que regresen a la comunidad de donde vinieron, como nos sugiere la literatura.

Para entender esta posición debemos señalar que las respuestas formales del sistema de justicia criminal hacia el delito, están basadas en varias ideologías que sirven para justificar el castigo. Estas vertientes reflejan las nociones que tiene la sociedad y aquellos que laboran dentro del sistema correccional, con respecto a la criminalidad, los criminales y la forma de tratarlos. Conklin (1989) identifica estas ideologías como: 
incapacitación, retribución y rehabilitación. En Puerto Rico, el discurso oficial hace hincapié en la filosofía de rehabilitación, pero en la práctica, se trabaja con las nociones de retribución o incapacitación. Congruentemente con ello, las personas participantes del grupo focal proveniente de Salud Correccional señalan que:

- "Todo es una excusa para la seguridad, coartando, engrilletando, esposando y reprimiendo."

- A las sumariadas se les tranca todo el día. Permanecen encerradas 24 horas, como a las de máxima".

- Salen cuando las saca la trabajadora o trabajador social, o cuando las llevan a bañarse. Estas mujeres son tratadas como a las confinadas de seguridad máxima. Se les vigila y se reprimen en extremo, lo cual las lleva a sentirse intimidadas por el sistema."

- "Con la excusa típica de la seguridad, se les escolta todo el tiempo y se les hace sentir intimidadas".

Las relaciones entre las confinadas y los oficiales de custodia (como agentes de control representativos de la Institución) es un tema que ocupó un punto central en la discusión entre las personas participantes del grupo focal de Salud Correccional. Consideran que los oficiales de custodia tratan con poco respeto a las confinadas:

- "Les hablan malo, les faltan el respeto y las intimidan."

- "Les crean confusión porque no las ayudan, las maltratan."

- "Las recriminan, las hacen sentir culpable, son jueces y sentencian."

- "Cuando ven una mujer que regresa a la Institución, la reciben con frases tales como: Tu otra vez aquí."

Más importante aún, para las participantes de este grupo focal es el 'asunto de la seguridad'. Se asume como instrumento punitivo y fiscalizador, añadiendo al maltrato que sufren las confinadas, en especial cuando transgreden alguna norma institucional. Señalan también que la sanción por estas violaciones se torna con frecuencia en un acto arbitrario del oficial de custodia: Expresaron que se utilizaba el control sobre las visitas para castigarlas y que sólo porque al Oficial de Custodia no le cae bien la confinada, le quita las visitas.

Bajo visiones divergentes y estereotipadas de la mujer confinada y el sistema carcelario, las personas participantes de los grupos focales identificaron las siguientes necesidades que para el servicio social que se les ofrece:

\section{A. Las necesidades más apremiantes}

La orientación legal es fundamental para todas las confinadas. Las expresiones del grupo de Salud Correccional fueron: 
- Las mujeres tienen poco o ningún acceso a orientación legal y no son partícipes del plan legal."

- $\quad$ No se les brinda orientación legal sobre la custodia de los hijos, derechos de vivienda, propiedad, entre otros. Hay muy pocas visitas de abogados a la Institución. El asesoramiento legal más común es: “¿Te haces culpable, o no?"

- "No hay un asesoramiento legal"

Tanto las Oficiales Socio- penales como las trabajadoras y trabajadores sociales y personal de Servicios de Salud Correccional reconocen, que el aspecto legal es el asunto más importante que condiciona la situación socio-emocional de las confinadas en la Institución. Entienden sin embargo, que las necesidades legales de las confinadas se definen por status, siendo las sumariadas las que más ayuda necesitan ya que muchas de ellas no tienen un abogado ó abogada que las asesore. Señalan: "La situación de las sumariadas es distinta a las sentenciadas, porque les crea tensión si no tienen abogado" (SSP).

Por su parte son las confinadas menores (16 a 21 años) las que más necesitan ayuda para mejorar sus condiciones de vida en la Institución y evitar el deterioro de su condición humana y la violación de derechos.

Para las menores no hay campamentos, ni servicios. Están en áreas cerradas, separada de los adultos, por razones de seguridad. No tienen tratamiento; nada vocacional. Ahora se está logrando que bajen a los talleres de cerámica. El problema con éstas jóvenes es el factor numérico, ya que son menos y esto les afecta para que se les asignen servicios (SSP).

Otras necesidades identificadas, son la orientación y ayuda socio-emocional para mejorar o re-establecer las relaciones familiares: En el grupo focal de socio-penales se señaló: "Desean estar con sus hijos". "Lo más que les preocupa es la comunicación con sus padres".

De la misma forma, surge la necesidad de vivienda. Esta se relaciona con la legislación de una falta y estás fuera'. Esta política conlleva el desahucio de la familia cuando un miembro es acusado por un delito relacionado con las drogas, o un crimen violento. Esto provoca incertidumbre ante la posibilidad de no tener alternativas de vivienda cuando egresen de la Institución. Ignorar esta situación en un plan de servicios es contrario a la meta personal e institucional de reintegro a la comunidad. En el plan de servicios que reflejan los expedientes revisados, esta situación se atiende con un referido a las agencias particulares como Departamento de la Vivienda, externalizando la situación sin seguimiento al respecto, ya que se considera como ajeno a sus funciones socio-penales. 


\section{B. $\quad$ Servicios y actividades}

El promedio de estadía de las confinadas en esta institución, basado en las sentencias, es de cinco años. Las condiciones de vida en la Institución serán vitales para su salud física y emocional como preparación para su re-ingreso a la comunidad. Un aspecto fundamental de las condiciones de vida durante su periodo carcelario, es identificado como la planta física. Ésta se describe como obsoleta, mal construida y mal distribuida. Algunas expresiones fueron:

- "Las facilidades físicas donde se ubican los servicios de Salud Correccional e ingresos de confinadas, están obsoletas."

"Las pacientes con condiciones crónicas están en edificios muy lejos del área médica y las oficinas para proveer el servicio no tiene aire acondicionado."

- $\quad$ "El espacio es insuficiente. El hospital se utiliza como facilidad de custodia máxima."

- "En el área del hospital, no hay agua caliente. Un baño para 20-25 confinadas."

- "El baño no tiene mezcladora, el agua sale a presión desde la pared" "En el área de admisiones no hay aire acondicionado" (SC).

Relacionado con esto, un aspecto sobresaliente mencionado en la discusión, fue la limitación en los servicios médicos especializados, para condiciones especiales. En los momentos en que se realizó el estudio no había personal de psiquiatría, ni dietista para atender las múltiples situaciones de salud mental o emocional causadas por la tensión de la vida carcelaria o las condiciones de salud que requieren alimentación especial tales como diabetes, embarazo o condiciones cardiacas. Tampoco contaban con los servicios prenatales, ni orientación, o educación para preparar a la mujer para el parto. Sobre todo, no se prepara a la mujer para la separación de su hijo ó hija cuando nazca. Además, en el grupo focal de Salud Correccional mencionaron: "Los servicios de CREA no son eficientes; son inconsistentes y condiciones como HIV y hepatitis, sin tratamiento."

El tema de las relaciones familiares como un elemento de apoyo para el proceso de vida en el encarcelamiento y la meta de reintegro a la comunidad fue importante dentro de la discusión con profesionales que participaron de los grupos focales. Estas personas expresaron que no se hacen acercamientos a familiares para informarles cuando la mujer confinada está enferma. La familia tampoco es incluida en el plan de servicios, ni de ayuda. La definición de familia es la tradicional, basada en lazos consanguíneos y legales, la cual no incluye amistades, compadrazgos, compañero o compañera consensual y por lo tanto, no se permiten estas visitas. Ello limita sustancialmente el número de recursos que pueden aportar para un plan de servicios de intervención social, al excluir otras personas significativas en la vida de cada confinada. Tampoco se 
reconoce como valioso que durante el periodo de institucionalización se desarrollen lazos de apego con familiares que estaban distanciados antes del confinamiento. En general, la familia no se ve como parte del servicio de trabajo social. Algunas de las expresiones en el grupo de Salud Correccional fueron:

- "No se fomenta la interacción con la familia."

- $\quad$ "No se les permite recibir la visita de los hijos e hijas que están bajo la custodia de personas o familias que no están relacionadas por lazos de consanguinidad."

- "No se considera familia a una pareja consensual o a amistades. Si no los identifican con nombre y apellido, no entran. Si la confinada tiene un familiar convicto, la comunicación es casi imposible".

De otra parte, aunque señalan la importancia de incluir a la familia como recurso para satisfacer las necesidades de las confinadas, también hacen hincapié en la responsabilidad institucional de satisfacer las mismas, ya que entienden que algunas no tienen recursos familiares que las ayuden: Una persona del grupo de Salud Correccional comentó: "Algunas no tienen familia, ni recursos para obtener ropa interior, ni del diario; en visita de familiares no se les permite traer ropa y los 'brassieres' no están permitidos por medidas de seguridad." Tampoco hay orientación sobre los procesos de vida en el confinamiento. La agencia tiene limitaciones para ofrecer los talleres educativos que son parte de las condiciones para que la confinada pueda egresar o acogerse a cualquier programa de libertad anticipada. Esto tiene repercusiones negativas para el proceso y status legal de las confinadas. Del mismo grupo focal mencionaron:

- $\quad$ "En ocasiones se le niega los privilegios en Corte, por no participar de servicios tales como Taller de Vida sin Violencia y otros, que la Institución, se supone que ofrezca y no ofrece."

- $\quad$ "Los traslados se hacen por consideraciones que nada tienen que ver con las necesidades de las confinadas".

Para atender estas situaciones, las personas participantes del grupo focal de Salud Correccional presentaron como alternativa la capacitación al personal de la Institución, sobre derechos legales humanos y sociales de las confinadas. Sobre todo, este debe ser prioritario para

Oficiales de Custodia. A pesar de que mencionan que se les provee adiestramiento, hacen hincapié en que el mismo no es suficiente.

Contrario a las expresiones anteriores, vertidas por el personal de Salud Correccional que participó del estudio, las técnicas de Servicios Socio- penales centraron su discusión en los servicios que ofrece la Institución como parte de los procesos de ‘rehabilitación': 
talleres y servicios técnicos para el desarrollo de destrezas vocacionales y de recreación tales como computación, cerámica y danza. Otros servicios que se identificaron son los siguientes: Hogares CREA, servicios de planificación familiar, área médica completa, estudios universitarios y otros servicios se dan a través de los programas de desvío. Aclararon que estos servicios son limitados, pero consideran que los mismos proveen alternativas para la rehabilitación aunque se den en el contexto de casos excepcionales y no de la norma: "Por ejemplo, una confinada que tomó todos los cursos y terapias de CREA, salió adelante" (SSP)

En relación con las confinadas adultas, sugieren que el enfoque principal debe estar en los programas vocacionales: "Programas que las ayuden a conseguir empleo y que ellas sepan a donde ir para continuar funcionando dentro de la comunidad" (SSP). La perspectiva de re-integro de la confinada a la comunidad estuvo ausente de la discusión en el grupo focal donde participaron las oficiales de servicios socio-penales.

\section{El trabajador y trabajadora social en las instituciones correccionales}

Un aspecto fundamental para esta discusión es que el Personal de Salud Correccional no se identifica como parte del sistema correccional. Su identidad profesional se deriva de su desempeño en un programa de apoyo a los servicios que ofrece a la confinada el sistema correccional. Las personas participantes de este grupo focal establecen que el rol principal de profesionales de trabajo social en este ambiente, es uno de psicoterapista, así como facilitador o facilitadora y enlace entre la Administración de Corrección y las necesidades de las mujeres confinadas, incluyendo la función de defensa de los derechos humanos y civiles de estas mujeres. Sin embargo, aparte de identificar esto como un rol importante, no se expresó cómo ello se manifiesta en los servicios que ofrecen. Un aspecto fundamental para su desempeño profesional, es que el mismo se desarrolla en un ambiente de muchas limitaciones de recursos como son: el que no tienen teléfono, ni aire acondicionado en las oficinas; tampoco tienen privacidad para que puedan atender a las confinadas y que estas confíen en el personal de ayuda.

Entre las dificultades señaladas en el grupo focal de Salud Correccional están las diferencias en la ejecución de sus funciones con la de los Oficiales de Custodia.

"Los Oficiales de Custodia toman ingerencia en todo."

"Es particularmente inquietante la interferencia en la privacidad de profesionales de trabajo social cuando están entrevistando."

"Los Oficiales de Custodia están presente en los procesos de entrevista y no permiten la confidencialidad y están pendientes de todo." "Cuando se les pide a los Oficiales de Custodia que hagan su trabajo fuera de la habitación, entonces asumen actitudes hostiles". 
Estas experiencias son cónsonas con hallazgos similares descritos por Coggins y Frezquez (2001), donde se señala que profesionales de trabajo social que laboran en instituciones carcelarias, pueden encontrar dificultades para que otros profesionales en la misma institución, entiendan los conceptos tales como confidencialidad y la necesidad de regirse por los códigos de ética profesional.

\section{Percepción de la función de las profesionales de Servicios Socio- penales}

En cuanto al personal de Servicios Socio-penales, este tema estuvo matizado por la circunstancia de que no son trabajadoras sociales. Como aclaración se señaló, que existe diversidad en el trasfondo académico para ocupar el puesto de técnica de servicios socio-penales. Ello incluye bachillerato en psicología, trabajo social y otras disciplinas relacionadas a las ciencias sociales. Su puesto de Técnica Socio-penal, lo definen como: "No somos trabajadoras sociales, somos técnicas de Servicios Socio-penales y eso te limita las funciones y te quedas llenando papeles. Haces tus funciones de trabajo social, pero existen distintas disciplinas dentro del trabajo de Técnicas de Servicios Socio-penales y por ello te quedas fuera del Colegio de Trabajadores Sociales, aunque ofrezcas los servicios." En cuanto a las funciones específicas, éstas establecen como premisa básica, que las mismas están determinadas por la estructura ocupacional de la agencia: "Las profesionales que trabajan aquí tienen que regirse por lo que determina la Administración de Corrección, o perder su título."

Esto evidencia la preocupación que presentan estas profesionales, al no tener el respaldo de un grupo profesional que les permita reclamar su espacio en el contexto laboral. Con poco poder de negociación, la agencia define su identidad profesional en la estructura de los servicios socio-penales que se ofrecen: "La función del Técnico de Servicios Socio-penales, se provee orientación, se llenan papeles, enlace con el Departamento de la Familia y agencias de gobierno sobre todo, ayudar a la confinada en su ajuste a la vida en la Institución" (SSP).

\section{Observaciones y Comentarios}

Ante los hallazgos presentados, los siguientes comentarios se dirigen hacia la meta institucional del regreso de estas mujeres a la comunidad, considerando lo que señalan Cook y Davies (1999), “... el arte de hacer tiempo bajo una orientación de sobrevivencia afirmativa y constructivamente" (pág. 17). En la institución carcelaria estudiada se observa una aplicación selectiva de los derechos de las confinadas como si fueran privilegios (las visitas de sus descendientes y familiares, negación de visitas por consideración de seguridad y otros). Ello constituye un ejercicio de poder dirigidos a la subordinación de la confinada, sin estrategias adecuadas para la rehabilitación. Como nos menciona Mary Jo, Betty y Tiki, confinadas de una institución penal para mujeres en Ohio en Watterson, (1996): 
Se comienza perdiendo la identidad propia tan pronto como se entra. Se deja de buscar o hacer cosas para una misma; pierdes interés en las cosas. Sientes que nada va a estar bien. Tienes que vestir en una sola forma, no es mucho lo que puedes hacer para ajustar tu uniforme; sólo puedes usar tacos hasta cierto nivel de alto y controlar el uso de maquillaje. Cuando comienzas a hacer trabajos que son para hombres, comienzas a sentir los problemas femeninos y sientes que se hieren tus sentimientos propios. (pág. 73)(Traducción por la autora)

Watterson (1996) concluye, que estas expresiones manifiestan la dependencia forzada que promueve el sistema carcelario. Para ella "la prisión es como un padre abusivo" (pág. 73). Al analizar esta situación, hay que considerar que muchas de estas mujeres han sido socialmente condicionadas a la dependencia y la subordinación, social, moral y económica. Confrontarlas nuevamente con el poder arbitrario y la dominación en las actividades Institucionales, es recrear las condiciones de violencia personal y estructural que las llevó a la cárcel. A pesar de que la violencia es parte de la cultura universal de la prisión (Cook y Davies, 1999), ésta se puede combatir con programas de educación al personal de la Institución para que se dirijan hacia la comprensión de la realidad, de que ambos grupos (confinadas y empleadas ó empleados) se encuentran inter-relacionados: por las circunstancias de la conducta delictiva de unas y las condiciones de trabajo de las otras. Esto debe ser el fundamento de una reflexión consciente hacia en cambio institucional que beneficie a todos y todas.

Un aspecto central de este cambio en los servicios socio-penales debe incluir la consideración de que la seguridad personal de la confinada: su vida, su salud física, mental y emocional, no va ha ser comprometida. Ello no se puede lograr sin un componente participativo en el que las confinadas tengan algún control sobre las normas, responsabilidades y beneficios o derechos, del entorno institucional que las cobija como residencia. Se debe garantizar la seguridad personal y emocional en contra de la violencia que pueda surgir de parte de otras confinadas y del personal de la Institución en general. Sobre ese particular, Cook y Davies (1999) señalan: "La necesidad de tener cierto grado de control sobre la vida propia, por más pequeño que sea, es parte fundamental de la naturaleza humana" (pág. 20).

El servicio que se brinda en las instituciones carcelarias para mujeres va encaminado, como se refleja en la discusión en los grupos focales participantes de este estudio, a la adaptación de la confinada al ambiente institucional. Se habla de rehabilitación y reintegro a la 'libre comunidad,' sin embargo, el modelo de rehabilitación que se sigue, es el de separar a la mujer de la comunidad y castigarla. La perdida de libertad es de por si la penalidad más grande que sufre una persona (Hoge, 2003). Por ello el modelo carcelario que limita y desalienta las relaciones personales y familiares, aísla aún más a esta mujer, de la comunidad a la que debe regresar en pocos años. Es importante re-enfocar el 'Plan de Servicios' para que se le brinde más atención a la 
identificación de recursos y a la creación y el fortalecimiento de grupos de apoyo mediante la coordinación inter-agencial. Es indispensable también, una mayor integración de los recursos familiares dentro de los procesos de ayuda que se le brinda en la misma Institución.

Uno de los aspectos más importante en los servicios socio-penales es la identificación del rol profesional del personal que los brinda. Fortalecer el conocimiento y apoyar al personal de servicios socio-penal y psico- social en su rol de intercesor, es esencial para garantizar los derechos humanos de las confinadas y proteger su ambiente institucional. Esta función ha sido reconocida como vital para la efectividad de los servicios, las metas y objetivos que estos programas pretenden. De la misma forma, establece una base para el reclamo de la acción participativa y democrática de estas mujeres. Esta conciencia debe transformarse en propuestas para cabildear activamente en los foros oficiales y de política pública, si es que se pretende promover cambios significativos en la agencia. La función de cabildeo mencionada como importante por profesionales de trabajo social y el personal psico- social de salud correccional, puede ser identificada en las palabras de María Stewart, mujer afro-americana del siglo diecinueve, quien entendía que el poder de auto definirse era esencial para la sobrevivencia de la mujer negra: "Demanden por sus derechos y privilegios. Conozcan las razones por las que no los pueden alcanzar. Provoquen preocupación con sus impertinencias. Usted puede morir si lo intenta; pero seguramente morirá si no lo hace." (Hill Collins, 2000, p.1)(Traducción por la autora)

Contrario a la creencia popular de que los ofensores son personas 'disfuncionales' social y psicológicamente, las cárceles no son ambientes designados únicamente para el control y el castigo de personas que han violado las leyes, si no ambientes donde una gran proporción de la población que allí se encuentra son capaces de la empatía hacia otros, y tienen deseo de mejorar personalmente (Coggins \& Fresquez, 2001). Por ello, el ambiente carcelario es el lugar donde pueden adquirir las destrezas sociales, emocionales, cognitivas e interpersonales que no tuvieron en el pasado.

\section{Conclusiones}

Uno de los hallazgos más sobresalientes de este análisis, es la diferencia sustancial en la visión que se tiene de la confinada y del servicio que se ofrece. Para Salud Correccional, la meta es el tratamiento y la prevención; para Corrección lo es la seguridad, la disciplina y el control de la confinada. Esta diferencia responde principalmente a la visión específica y las expectativas programáticas de ambos grupos. Ello puede ser también, producto de la fragmentación administrativa y de programas de bienestar social que existe en la Agencia y en la comunidad en general.

Otro asunto relevante es la falta de poder de su rol profesional ante las metas y agenda de control que sirve de base al modelo de servicio del sistema carcelario. El rol de 
trabajo social y los servicios socio penales designado por la Institución, es contribuir a la agenda de penalización de la mujer confinada y a su adaptación al ambiente carcelario. Se promueve la dependencia y no la independencia emocional, física o económica. El reto de los trabajadores sociales y del personal de servicios sociopenales es por lo tanto, el rescate de los valores intrínsecos de su profesión para promover la acción ético política que les corresponde, en favor de cambios institucionales para que estos redunden en un servicio donde el tiempo de encarcelación sea un modelo para 'hacer tiempo constructivamente'. 


\section{Referencias}

Cámara de Representantes de Puerto Rico (30 de enero de 1997), $R$ de la C. 130 del 22 de enero de 1997. Texto de aprobación final por la Cámara.

Conkling, John, E. (1989). Criminology. (3er. ed.). New York: MacMillan Pub. Co.

Coggins, Kip; Fresquez, J. Eli (2001). Working with Clients in Correctional Settings: A guide for social workers and Corrections Professionals. Iowa: Eddie Bowers Publishing, Inc.

Cook, Sandy; Davies, Susanne (1999). Harsh Punishment: International Experiences of women's imprisonment. Boston: Northeastern University Press.

Departamento de Corrección y Rehabilitación de Puerto Rico. (2005). Perfil de la mujer confinada. San Juan, P R: Autor.

González Rodríguez, Emilio; Márquez Ramos, Aida W. y Márquez Rosario, Dixie (2003). Relaciones materno filiales de las confinadas: La Satisfacción de la Madre Confinada en la Escuela Industrial para Mujeres de Vega Alta con los Sistemas de Apoyo del Departamento de la Familia, la Institución Carcelaria y sus Familiares para Mantener la Relación Interno Filiar, los Medios de Comunicación y el Conocimiento de la Madre sobre las Experiencias Cotidianas de sus Hijos a Mayo de 2003. Tesis de maestría no publicada. Escuela Graduada de Trabajo Social, Universidad de Puerto Rico en Río Piedras.

Harden, Judy \& Hill, Marcia (Eds) (1998). Breaking the rules: Women in prison and femenist therapy. New York: The Haworth Press, Inc.

Hill Collins, Patricia. (2000). Black Feminist Thought: Knowledge, Conciousness, and the Politics of Empowerment._( $2^{\text {nd }}$ ed.). New York: Routledge.

Hoge, Warren (2003). Finish Prison: No Gates or Armed Guards. Recuperado el 03/ 01/04 en: www.nytimes.com/2003/01/02/ international/europe/02FINL.

Contextual Framework. En:Judy Harden y Marcia Hill,(Eds) (1998). Breaking the rules: Women in prison and femenist therapy. New York: The Haworth Press, Inc.

Picó, Fernando (1994). El Día Menos Pensado: Historia de los Presidiarios en Puerto Rico (1793- 1993). San Juan, PR: Ediciones Huracán.

Rose and Associates (N/D). Female Offenders Profile. Mayaguez, PR: Autor. 
Smart, Carol (1995). Law, crime and sexuality. Los Angeles: Sage Publications.

Watterson, Kathryn (1996). Women in Prison: Inside the Concrete Womb. (Second Edition). Boston: Northeastern University Press.

\section{Reconocimiento}

Agradezco la participación del personal del programa de Salud Correccional y Servicios Socio-penales en las actividades que permitieron completar este estudio. También agradezco a la Administración de la Escuela Industrial Para Mujeres en Vega Alta por permitir y facilitar el acceso a la Institución. De la misma forma mi agradecimiento a los compañeros del proyecto de investigación sobre el Perfil de la Mujer Confinada auspiciada por el Instituto FILIUS (Institute of Correction and Rehabilitation Central Administration) por su colaboración y apoyo en las distintas fases de este proyecto. 